\title{
Tree-loop duality relation beyond single poles
}

\section{Isabella Bierenbaum, ${ }^{a}$ Sebastian Buchta, ${ }^{b}$ Petros Draggiotis, ${ }^{b}$ Ioannis Malamos ${ }^{b}$ and Germán Rodrigo ${ }^{b}$}

\author{
${ }^{a}$ II. Institut für Theoretische Physik, Universität Hamburg, \\ Luruper Chaussee 149, 22761, Hamburg, Germany \\ ${ }^{b}$ Instituto de Física Corpuscular, Universitat de València, \\ Consejo Superior de Investigaciones Cientificas, \\ Parc Cientific, E-46980 Paterna (Valencia), Spain \\ E-mail: isabella.bierenbaum@desy.de, sbuchta@ific.uv.es, \\ petros.drangiotis@ific.uv.es, ioannis.malamos@ific.uv.es, \\ german.rodrigo@ific.uv.es
}

Abstract: We develop the Tree-Loop Duality Relation for two- and three-loop integrals with multiple identical propagators (multiple poles). This is the extension of the Duality Relation for single poles and multi-loop integrals derived in previous publications. We prove a generalization of the formula for single poles to multiple poles and we develop a strategy for dealing with higher-order pole integrals by reducing them to single pole integrals using Integration By Parts.

KeYwords: QCD Phenomenology, NLO Computations

ArXiv EPRINT: 1211.5048 


\section{Contents}

1 Introduction 1

2 Duality relation at one- and two-loops 2

$\begin{array}{lll}3 & \text { Duality relation for multiple poles } & 7\end{array}$

4 Reducing to single poles with IBPs $\quad 10$

$\begin{array}{ll}4.1 \text { The case for two-loop diagrams } & 12\end{array}$

$\begin{array}{ll}4.2 \text { The case for three-loop diagrams } & 16\end{array}$

$\begin{array}{lll}5 & \text { Conclusions } & 19\end{array}$

$\begin{array}{ll}\text { A Proof of the reduction of eq. (4.6) } & 20\end{array}$

\section{Introduction}

The last 15 years have seen extraordinary progress in the analytical and numerical computation of cross sections in the Standard Model at one- and two-loops and even higher orders. Once considered the bottleneck of numerical applications, higher- than-tree-level corrections exhibit high complexity, partly due to the enormous number of Feynman diagrams needed, sometimes numbered in hundreds or even thousands, for important cross sections. Important efforts have been devoted to developing efficient methods able to boost forward the calculational capability both at the multi-leg and the multi-loop frontier. Today, $2 \rightarrow 4$ processes at next-to-leading order (NLO), either from Unitarity based methods, [1-3], or from a more traditional Feynman diagrammatic approach, [4], are affordable and are even becoming standardized. There has also been a lot of progress concerning next-to-next-to leading order (NNLO) calculations [5-16].

The importance of higher order corrections cannot be overstated. While leading-order (LO) predictions of multi-particle processes at hadron colliders in perturbative Quantum Chromodynamics (pQCD) provide, in general, a rather poor description of experimental data, NLO is the first order at which normalizations, and in some cases, the shapes, of cross sections can be considered reliable [17]. NNLO, besides improving the determination of normalizations and shapes, is also generally accepted to provide the first serious estimate of the theoretical uncertainty in pQCD. Despite the relatively smaller coupling, electroweak (EW) radiative NLO corrections might also be sizable at the LHC, [18, 19]. Precision theoretical predictions for background and signal multi-particle hard scattering processes, in the SM and beyond, are mandatory for the phenomenological interpretation of experimental data, and thus to achieve a successful exploitation of the LHC physics programme. 
In [20], a novel method was developed for the calculation of multileg one-loop amplitudes. Called the Duality Theorem, it applies directly the Cauchy Residue Theorem to one-loop integrals. The result can be represented by single cuts to Feynman diagrams, integrated over a modified phase space. (Note also [21] where the author uses retarded boundary conditions to obtain a duality between loop and tree graphs). The Duality Theorem was extended in [23] beyond the one-loop level, to two- and three-loops and it was shown how it can be extended to an arbitrary number of loops. The main feature and advantage of this approach is that at any number of loops, an amplitude can be written as a sum of tree-level objects, obtained after making all possible cuts to the lines of a Feynman diagram, one cut per loop and integrated over a measure that closely resembles the phase space of the corresponding real corrections. This modified phase-space, raises the intriguing possibility that virtual and real corrections can be brought together under a common integral and treated with Monte-Carlo techniques at the same time. In these papers the Duality Theorem was developed for diagrams and their integrals, that do not possess identical propagators. This possibility does not appear at one-loop for a convenient choice of gauge [20], but it is always present for higher order corrections. Identical propagators possess higher than single poles and the Duality Theorem developed so far, which is based on the Cauchy formula for single poles, must be extended to accomodate for this new feature.

The purpose of this work is to extend the Duality Theorem to graphs and integrals with multiple poles at two- and three-loops and to present a procedure, a strategy for dealing with higher poles in an amplitude calculation that retains the features and advantages of the Duality theorem as detailed in [23]. The paper is organized as follows: in section 2 we recall the basic definitions and results concerning the Duality Theorem at one- and two-loops and beyond, for integrals with single poles. In section 3 we derive in detail the extension of the Duality Theorem to integrals with double and multiple propagators, which exhibit multiple poles in the complex plane. In section 4 we present an alternative method to deal with multiple poles at two- and three-loops which retains the basic advantages of the Duality Theorem. It is based on Integration By Parts relations that allow to rewrite integrals with multiple poles in terms of integrals involving only single poles.

\section{Duality relation at one- and two-loops}

A general one-loop $N$-leg, scalar integral, such as the one shown in figure 1, is written as:

$$
L^{(1)}\left(p_{1}, p_{2}, \ldots, p_{N}\right)=\int_{\ell_{1}} \prod_{i=1}^{N} G_{F}\left(q_{i}\right),
$$

where

$$
G_{F}\left(q_{i}\right)=\frac{1}{q_{i}^{2}-m_{i}^{2}+i 0}
$$

is the Feynman propagator. The four-momenta of the external legs are denoted $p_{i}, i=$ $\{1,2, \ldots N\}$. All are taken as outgoing and ordered clockwise. The loop momentum is $\ell_{1}$, which flows anti-clockwise. The momenta of the internal lines $q_{i}$, are defined as

$$
q_{i}=\ell_{1}+p_{1, i}, \quad i \in \alpha_{1}=\{1,2, \ldots N\}, \quad p_{1, i}=p_{1}+\ldots+p_{i} .
$$


Throughout this paper we use the short-hand notation

$$
\widetilde{\delta}\left(q_{i}\right) \equiv 2 \pi i \theta\left(q_{i, 0}\right) \delta\left(q_{i}^{2}-m_{i}^{2}\right)=2 \pi i \delta_{+}\left(q_{i}^{2}-m_{i}^{2}\right), \quad \int_{\ell_{i}} \bullet=-i \int \frac{d^{d} \ell_{i}}{(2 \pi)^{d}} \bullet
$$

where the subscript + of $\delta_{+}$refers to the on-shell mode with positive definite energy, $q_{i, 0} \geq 0$. Hence, the phase-space integral of a physical particle with momentum $q_{i}$, i.e., an on-shell particle with positive-definite energy, $q_{i}^{2}=m_{i}^{2}, q_{i, 0} \geq 0$, reads:

$$
\int \frac{d^{d} q_{i}}{(2 \pi)^{d-1}} \theta\left(q_{i, 0}\right) \delta\left(q_{i}^{2}-m_{i}^{2}\right) \cdots \equiv \int_{q_{i}} \widetilde{\delta}\left(q_{i}\right) \cdots .
$$

It was shown in $[20,23]$ that using the Cauchy residue theorem the one-loop integral can be written in the form:

$$
L^{(1)}\left(p_{1}, p_{2}, \ldots, p_{N}\right)=-\sum_{i} \int_{\ell_{1}} \widetilde{\delta}\left(q_{i}\right) \prod_{\substack{j=1 \\ j \neq i}}^{N} G_{D}\left(q_{i} ; q_{j}\right)
$$

where

$$
G_{D}\left(q_{i} ; q_{j}\right)=\frac{1}{q_{j}^{2}-m_{j}^{2}-i 0 \eta\left(q_{j}-q_{i}\right)}
$$

is the so-called dual propagator, as defined in ref. [20], with $\eta$ a future-like vector,

$$
\eta_{0} \geq 0, \eta^{2}=\eta_{\mu} \eta^{\mu} \geq 0
$$

i.e., a $d$-dimensional vector that can be either light-like $\left(\eta^{2}=0\right)$ or time-like $\left(\eta^{2}>0\right)$ with positive definite energy $\eta_{0}$. The result in eq. (2.6), contrary to the Feynman-Tree theorem (FTT) $[25,26]$, contains only single-cut integrals. Multiple-cut integrals, like those that appear in the FTT, are absent thanks to modifying the original $+i 0$ prescription of the uncut Feynman propagators by the new prescription $-i 0 \eta\left(q_{j}-q_{i}\right)$, which is named the 'dual' $i 0$ prescription or, briefly, the $\eta$ prescription. The dual $i 0$ prescription arises from the fact that the original Feynman propagator $G_{F}\left(q_{j}\right)$ is evaluated at the complex value of the loop momentum $\ell_{1}$, which is determined by the location of the pole at $q_{i}^{2}-m_{i}^{2}+i 0=0$. The $i 0$ dependence of the pole of $G_{F}\left(q_{i}\right)$ modifies the $i 0$ dependence in the Feynman propagator $G_{F}\left(q_{j}\right)$, leading to the total dependence as given by the dual $i 0$ prescription. The presence of the vector $\eta_{\mu}$ is a consequence of using the residue theorem and the fact that the residues at each of the poles are not Lorentz-invariant quantities. The Lorentz-invariance of the loop integral is recovered after summing over all the residues. Furthermore, in the one-loop case, the momentum difference $\eta\left(q_{j}-q_{i}\right)$ is independent of the integration momentum $\ell_{1}$, and only depends on the momenta of the external legs (cf. eq. (2.3)).

The extension of the Duality Theorem to two loops has been discussed in detail in $[23,24]$. Here we recall the basic points. We extend the definition of propagators of single momenta to combinations of propagators of sets of internal momenta. Let $\alpha_{k}$ be any set of internal momenta $q_{i}, q_{j}$ with $i, j \in \alpha_{k}$. We then define Feynman and dual propagator functions of this set $\alpha_{k}$ in the following way:

$$
G_{F}\left(\alpha_{k}\right)=\prod_{i \in \alpha_{k}} G_{F}\left(q_{i}\right), \quad G_{D}\left(\alpha_{k}\right)=\sum_{i \in \alpha_{k}} \widetilde{\delta}\left(q_{i}\right) \prod_{\substack{j \in \alpha_{k} \\ j \neq i}} G_{D}\left(q_{i} ; q_{j}\right) .
$$




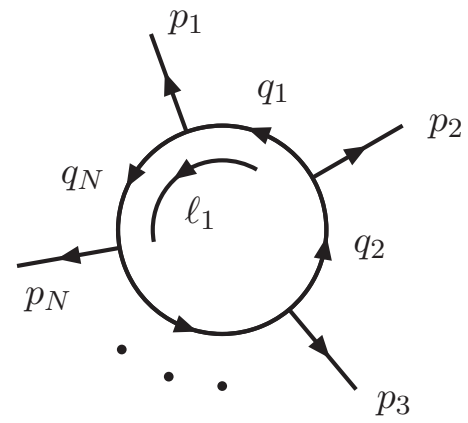

Figure 1. Momentum configuration of the one-loop $N$-point scalar integral.

By definition, $G_{D}\left(\alpha_{k}\right)=\widetilde{\delta}\left(q_{i}\right)$, when $\alpha_{k}=\{i\}$ and thus consists of a single four momentum. At one-loop order, $\alpha_{k}$ is naturally given by all internal momenta of the diagram which depend on the single integration loop momentum $\ell_{1}, \alpha_{k}=\{1,2, \ldots, N\}$. However, let us stress that $\alpha_{k}$ can in principle be any set of internal momenta. At higher order loops, e.g., several integration loop momenta are needed, and we can define several loop lines $\alpha_{k}$ to label all the internal momenta (cf. eq. (2.19)) where eq. (2.9) will be used for these loop lines or unifications of these. We also define:

$$
G_{D}\left(-\alpha_{k}\right)=\sum_{i \in \alpha_{k}} \widetilde{\delta}\left(-q_{i}\right) \prod_{\substack{j \in \alpha_{k} \\ j \neq i}} G_{D}\left(-q_{i} ;-q_{j}\right),
$$

where the sign in front of $\alpha_{k}$ indicates that we have reversed the momentum flow of all the internal lines in $\alpha_{k}$. For Feynman propagators, moreover, $G_{F}\left(-\alpha_{k}\right)=G_{F}\left(\alpha_{k}\right)$. Using this notation the following relation holds for any set of internal momenta $\alpha_{k}$ :

$$
G_{A}\left(\alpha_{k}\right)=G_{F}\left(\alpha_{k}\right)+G_{D}\left(\alpha_{k}\right),
$$

where $G_{A}\left(q_{i}\right)$ is the advanced propagator:

$$
G_{A}\left(q_{i}\right)=\frac{1}{q_{i}^{2}-m_{i}^{2}-i 0 q_{i, 0}},
$$

and

$$
G_{A}\left(\alpha_{k}\right)=\prod_{i \in \alpha_{k}} G_{A}\left(q_{i}\right)
$$

The proof of eq. (2.11) can be found in ref. [23]. Note that individual terms in $G_{D}\left(\alpha_{k}\right)$ depend on the dual vector $\eta$, but the sum over all terms contributing to $G_{D}\left(\alpha_{k}\right)$ is independent of it. Another crucial relation for the following is given by a formula that allows to express the dual function of a set of momenta in terms of chosen subsets. Considering the following set $\beta_{N} \equiv \alpha_{1} \cup \ldots \cup \alpha_{N}$, where $\beta_{N}$ is the unification of various subsets $\alpha_{i}$, we can obtain the relation:

$$
G_{D}\left(\alpha_{1} \cup \alpha_{2} \cup \ldots \cup \alpha_{N}\right)=\sum_{\beta_{N}^{(1)} \cup \beta_{N}^{(2)}=\beta_{N}} \prod_{i_{1} \in \beta_{N}^{(1)}} G_{D}\left(\alpha_{i_{1}}\right) \prod_{i_{2} \in \beta_{N}^{(2)}} G_{F}\left(\alpha_{i_{2}}\right) .
$$




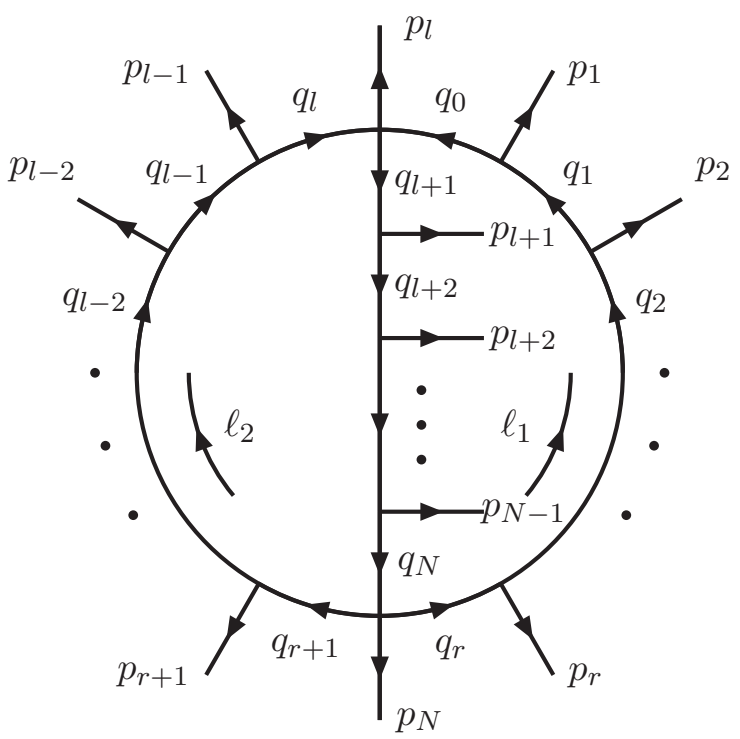

Figure 2. Momentum configuration of the two-loop $N$-point scalar integral.

The sum runs over all partitions of $\beta_{N}$ into exactly two blocks $\beta_{N}^{(1)}$ and $\beta_{N}^{(2)}$ with elements $\alpha_{i}$,

$i \in\{1, \ldots, N\}$, where, contrary to the usual definition, we include the case: $\beta_{N}^{(1)} \equiv \beta_{N}$, $\beta_{N}^{(2)} \equiv \emptyset$. For the case of $N=2$, e.g., where $\beta_{2} \equiv \alpha_{1} \cup \alpha_{2}$, we have:

$$
G_{D}\left(\alpha_{1} \cup \alpha_{2}\right)=G_{D}\left(\alpha_{1}\right) G_{D}\left(\alpha_{2}\right)+G_{D}\left(\alpha_{1}\right) G_{F}\left(\alpha_{2}\right)+G_{F}\left(\alpha_{1}\right) G_{D}\left(\alpha_{2}\right) .
$$

Naturally it holds that:

$$
G_{F}\left(\alpha_{1} \cup \alpha_{2} \cup \ldots \cup \alpha_{N}\right)=\prod_{i=1}^{N} G_{F}\left(\alpha_{i}\right) .
$$

Since in general relation (2.14) holds for any set of basic elements $\alpha_{i}$ which are sets of internal momenta, one can look at these expressions in different ways, depending on the given sets and subsets considered. If we define, for example, the basic subsets $\alpha_{i}$ to be given by single momenta $q_{i}$, and since in that case $G_{D}\left(q_{i}\right)=\widetilde{\delta}\left(q_{i}\right)$, eq. (2.14) then denotes a sum over all possible differing m-tuple cuts for the momenta in the set $\beta_{N}$, while the uncut propagators are Feynman propagators. These cuts start from single cuts up to the maximal number of cuts given by the term where all the propagators of the considered set are cut. Using this notation, the Duality Theorem at one-loop can be written in the compact form:

$$
L^{(1)}\left(p_{1}, p_{2}, \ldots, p_{N}\right)=-\int_{\ell_{1}} G_{D}\left(\alpha_{1}\right),
$$

where $\alpha_{1}$ as in eq. (2.3) labels all internal momenta $q_{i}$. In this way, we directly obtain the duality relation between one-loop integrals and single-cut phase-space integrals and hence eq. (2.17) can also be interpreted as the application of the Duality Theorem to the given set of momenta $\alpha_{1}$. It obviously agrees, at one loop, with eq. (2.6). 
We now turn to the general two-loop master diagram, as presented in figure 2. Again, all external momenta $p_{i}$ are taken as outgoing, and we have $p_{i, j}=p_{i}+p_{i+1}+\ldots+p_{j}$, with momentum conservation $p_{1, N}=0$. The label $i$ of the external momenta is defined modulo $N$, i.e., $p_{N+i} \equiv p_{i}$. In the two-loop case, unlike at the one-loop order, the number of external momenta might differ from the number of internal momenta. The loop momenta are $\ell_{1}$ and $\ell_{2}$, which flow anti-clockwise and clockwise respectively. The momenta of the internal lines are denoted by $q_{i}$ and are explicitly given by

$$
q_{i}= \begin{cases}\ell_{1}+p_{1, i} & , i \in \alpha_{1} \\ \ell_{2}+p_{i, l-1} & , i \in \alpha_{2} \\ \ell_{1}+\ell_{2}+p_{i, l-1} & , i \in \alpha_{3},\end{cases}
$$

where $\alpha_{k}$, with $k=1,2,3$, are defined as the set of lines, propagators respectively, related to the momenta $q_{i}$, for the following ranges of $i$ :

$$
\alpha_{1} \equiv\{0,1, \ldots, r\}, \quad \alpha_{2} \equiv\{r+1, r+2, \ldots, l\}, \quad \alpha_{3} \equiv\{l+1, l+2, \ldots, N\} .
$$

In the following, we will use $\alpha_{k}$ for denoting a set of indices or the set of the corresponding internal momenta synonymously. Furthermore, we will refer to these lines often simply as the "loop lines".

We shall now extend the duality theorem to the two-loop case, by applying eq. (2.17) iteratively. We consider first, in the most general form, a set of several loop lines $\alpha_{1}$ to $\alpha_{N}$ depending on the same integration momentum $\ell_{i}$, and find

$$
\int_{\ell_{i}} G_{F}\left(\alpha_{1} \cup \alpha_{2} \cup \ldots \cup \alpha_{N}\right)=-\int_{\ell_{i}} G_{D}\left(\alpha_{1} \cup \alpha_{2} \cup \ldots \cup \alpha_{N}\right),
$$

which states the application of the duality theorem, eq. (2.17), to the set of loop lines belonging to the same loop. Eq. (2.20) is the generalization of the Duality Theorem found at one-loop to a single loop of a multi-loop diagram. Each subsequent application of the Duality Theorem to another loop of the same diagram will introduce an extra single cut, and by applying the Duality Theorem as many times as the number of loops, a given multi-loop diagram will be opened to a tree-level diagram. The Duality Theorem, eq. (2.20), however, applies only to Feynman propagators, and a subset of the loop lines whose propagators are transformed into dual propagators by the application of the Duality Theorem to the first loop might also be part of the next loop (cf., e.g., the "middle" line belonging to $\alpha_{3}$ in figure 2). The dual function of the unification of several subsets can be expressed in terms of dual and Feynman functions of the individual subsets by using eq. (2.14) (or eq. (2.15)) though, and we will use these expressions to transform part of the dual propagators into Feynman propagators, in order to apply the Duality Theorem to the second loop. Therefore, applying eq. (2.20) to the loop with loop momentum $\ell_{1}$, reexpressing the result via eq. (2.15) in terms of dual and Feynman propagators and applying eq. (2.20) to the second loop with momentum $\ell_{2}$, we obtain the duality relation at two loops in the form:

$$
\begin{aligned}
L^{(2)} & \left(p_{1}, p_{2}, \ldots, p_{N}\right) \\
& =\int_{\ell_{1}} \int_{\ell_{2}}\left\{-G_{D}\left(\alpha_{1}\right) G_{F}\left(\alpha_{2}\right) G_{D}\left(\alpha_{3}\right)+G_{D}\left(\alpha_{1}\right) G_{D}\left(\alpha_{2} \cup \alpha_{3}\right)+G_{D}\left(\alpha_{3}\right) G_{D}\left(-\alpha_{1} \cup \alpha_{2}\right)\right\} .
\end{aligned}
$$


This is the dual representation of the two-loop scalar integral as a function of double-cut integrals only, since all the terms of the integrand in eq. (2.21) contain exactly two dual functions as defined in eq. (2.9). The integrand in eq. (2.21) can then be reinterpreted as the sum over tree-level diagrams integrated over a two-body phase-space.

The integrand in eq. (2.21), however, contains several dual functions of two different loop lines, and hence dual propagators whose dual $i 0$ prescription might still depend on the integration momenta. This is the case for dual propagators $G_{D}\left(q_{i} ; q_{j}\right)$ where each of the momenta $q_{i}$ and $q_{j}$ belong to different loop lines. If both momenta belong to the same loop line the dependence on the integration momenta in $\eta\left(q_{j}-q_{i}\right)$ obviously cancels, and the complex dual prescription is determined by external momenta only. The dual prescription $\eta\left(q_{j}-q_{i}\right)$ can thus, in some cases, change sign within the integration volume, therefore moving up or down the position of the poles in the complex plane. To avoid this, we should reexpress the dual representation of the two-loop scalar integral in eq. (2.21) in terms of dual functions of single loop lines. This transformation was unnecessary at oneloop because at the lowest order all the internal momenta depend on the same integration loop momenta; in other words, there is only a single loop line.

Inserting eq. (2.15) in eq. (2.21) and reordering some terms, we arrive at the following representation of the two-loop scalar integral

$$
\begin{aligned}
& L^{(2)}\left(p_{1}, p_{2}, \ldots, p_{N}\right) \\
& =\int_{\ell_{1}} \int_{\ell_{2}}\left\{G_{D}\left(\alpha_{1}\right) G_{D}\left(\alpha_{2}\right) G_{F}\left(\alpha_{3}\right)+G_{D}\left(-\alpha_{1}\right) G_{F}\left(\alpha_{2}\right) G_{D}\left(\alpha_{3}\right)+G^{*}\left(\alpha_{1}\right) G_{D}\left(\alpha_{2}\right) G_{D}\left(\alpha_{3}\right)\right\},
\end{aligned}
$$

where

$$
G^{*}\left(\alpha_{1}\right) \equiv G_{F}\left(\alpha_{1}\right)+G_{D}\left(\alpha_{1}\right)+G_{D}\left(-\alpha_{1}\right) .
$$

In eq. (2.22), the $i 0$ prescription of all the dual propagators depends on external momenta only. Through eq. (2.23), however, eq. (2.22) contains also triple cuts, given by the contributions with three $G_{D}\left(\alpha_{k}\right)$. The triple cuts are such that they split the two-loop diagram into two disconnected tree-level diagrams. By definition, however, the triple cuts are such that there is no more than one cut per loop line $\alpha_{k}$. Since there is only one loop line at one-loop, it is also clear why we did not generate disconnected graphs at this loop order. For a higher number of loops, we expect to find at least the same number of cuts as the number of loops, and topology-dependent disconnected tree diagrams built by cutting up to all the loop lines $\alpha_{k}$. These results can be generalized at three-loops and beyond without any additional effort. The reader is refered to [23] for further details.

\section{Duality relation for multiple poles}

In the previous section we applied the Residue Theorem to one- and two-loop graphs that contain only single poles, i.e. no identical propagators. At one-loop this is always the case for a convenient choice of gauge [20]. However, at higher loops there exists the possibility of identical propagators, i.e. higher order poles. Obviously, we need to generalize the Duality 


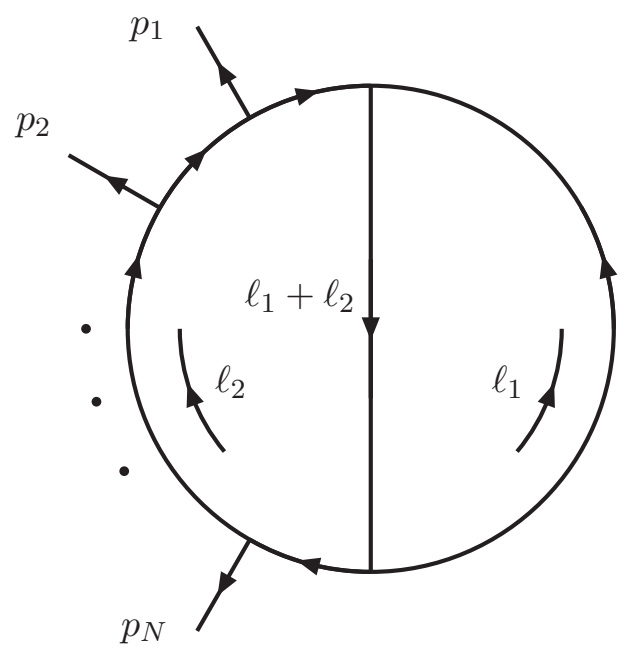

Figure 3. The two-loop $N$-point scalar integral with a double pole.

Theorem to accomodate for such graphs. The first occurence of higher order poles is at the two-loop level, with the sole double pole generic graph shown in figure 3 . The Duality Theorem can be derived for such graphs as well, using the Residue Theorem for multiple poles

$$
\operatorname{Res}_{\left\{z=z_{0}\right\}} f(z)=\left.\frac{1}{(k-1) !}\left(\frac{d^{k-1}}{d z^{k-1}}\left(z-z_{0}\right)^{k} f(z)\right)\right|_{z=z_{0}} .
$$

The derivation follows similar steps as with the single pole case and is independent of any particular coordinate system. We will derive an expression both in cartesian and light-cone coordinates, to demonstrate this independence. We start with the cartesian system. We write the Feynman propagator in a form that makes the poles explicit, i.e,

$$
G_{F}\left(q_{i}\right)=\frac{1}{\left(q_{i 0}-q_{i 0}^{(+)}\right)\left(q_{i 0}+q_{i 0}^{(+)}\right)}
$$

where $q_{i 0}^{(+)}=\sqrt{\mathbf{q}_{i}^{2}+m_{i}^{2}-i 0}$ is the position of the pole. Then, applying the Residue Theorem by selecting poles with negative imaginary part, we have

$$
\operatorname{Res}_{\left\{\operatorname{Im} q_{i 0}<0\right\}} G_{F}^{2}\left(q_{i}\right)=-\frac{2}{\left(2 q_{i 0}^{(+)}\right)^{3}}=-\int d q_{0} \frac{1}{2\left(q_{i 0}^{(+)}\right)^{2}} \delta_{+}\left(q_{i}^{2}-m_{i}^{2}\right) .
$$

The imaginary component of the new denominator $1 / 2\left(q_{i 0}^{(+)}\right)^{2}$ is irrelevant, because it is always a positive quantity. We refer the reader to [20] where the calculation for the case of simple poles is explained in more detail. Then, we assume the following Lorentz-invariant prescription of the residue

$$
\operatorname{Res}_{\left\{\operatorname{Im} q_{i 0}<0\right\}} G_{F}^{2}\left(q_{i}\right)=-\int d q_{0} \frac{\eta^{2}}{2\left(\eta q_{i}\right)^{2}} \delta_{+}\left(q_{i}^{2}-m_{i}^{2}\right),
$$


where $\eta^{\mu}=\left(\eta_{0}, \mathbf{0}\right)$ is a future-like vector, $\eta_{0}>0$, in cartesian coordinates. Contrary to the one loop case, where numerators depending on the loop momentum do not modify the duality prescription, in the two loop and higher orders cases the derivative in the residue calculation introduced by the higher order poles act on every single term in the numerator and also on the remaining propagators. Let $N\left(\alpha_{k}\right)$ be a function of a set of momenta $q_{l}$, with $l \in \alpha_{k}$. Then the residue of a double pole is given by

$$
\begin{aligned}
\operatorname{Res}_{\left\{\operatorname{Im} q_{i 0}<0\right\}} & \left\{G_{F}^{2}\left(q_{i}\right)\left(\prod_{j \neq i} G_{F}\left(q_{j}\right)\right) N\left(\alpha_{k}\right)\right\}=\left.\frac{\partial}{\partial q_{0}} \frac{1}{\left(q_{i 0}+q_{i 0}^{(+)}\right)^{2}}\left(\prod_{j \neq i} G_{F}\left(q_{j}\right)\right) N\left(\alpha_{k}\right)\right|_{q_{i 0}=q_{i 0}^{(+)}} \\
= & \left(\prod_{j \neq i} G_{D}\left(q_{i} ; q_{j}\right)\right) \frac{1}{\left(2 q_{i 0}^{(+)}\right)^{2}}\left[-\frac{1}{q_{i 0}^{(+)}}-\sum_{j \neq i}\left(2 q_{j 0}\right) G_{D}\left(q_{i} ; q_{j}\right)+\frac{\partial}{\partial q_{0}}\right] N\left(\alpha_{k}\right),
\end{aligned}
$$

which can be written as

$$
\begin{array}{r}
\operatorname{Res}\left\{\operatorname{Im} q_{i 0}<0\right\} \\
\left\{G_{F}^{2}\left(q_{i}\right)\left(\prod_{j \neq i} G_{F}\left(q_{j}\right)\right) N\left(\alpha_{k}\right)\right\}=\int d q_{0} \delta_{+}\left(q_{i}^{2}-m_{i}^{2}\right)\left(\prod_{j \neq i} G_{D}\left(q_{i} ; q_{j}\right)\right) \\
\times\left[-\frac{\eta^{2}}{2\left(\eta q_{i}\right)^{2}}-\sum_{j \neq i} \frac{\eta q_{j}}{\eta q_{i}} G_{D}\left(q_{i} ; q_{j}\right)+\frac{1}{2 \eta q_{i}} \frac{\partial}{\partial \eta q_{i}}\right] N\left(\alpha_{k}\right) .
\end{array}
$$

In light-cone coordinates we choose our coordinates such that in the plus component complex plane the poles with negative imaginary part are located at:

$$
q_{i+}^{(+)}=\frac{q_{i \perp}^{2}+m_{i}^{2}-i 0}{2 q_{i-}}, \quad \text { with } \quad q_{i-}>0 .
$$

In these light cone coordinates the Feynman propagator reads:

$$
G_{F}\left(q_{i}\right)=\frac{1}{2 q_{i-}\left(q_{i+}-q_{i+}^{(+)}\right)},
$$

and thus

$$
\operatorname{Res}_{\left\{\operatorname{Im} q_{i 0}<0\right\}} \theta\left(q_{i-}\right) G_{F}^{2}\left(q_{i}\right)=0,
$$

which, at first sight, seems to contradict equation eq. (3.4). This contradiction can be resolved by taking into account the fact that in light cone coordinates, the dual vector $\eta$ is lightlike and therefore $\eta^{2}=0$. Hence equation eq. (3.4) remains valid. Now, we are ready to calculate the residue of a double pole in light cone coordinates:

$$
\begin{gathered}
\operatorname{Res}_{\left\{\operatorname{Im} q_{i 0}<0\right\}}\left\{\theta\left(q_{i-}\right) G_{F}^{2}\left(q_{i}\right)\left(\prod_{j \neq i} G_{F}\left(q_{j}\right)\right) N\left(\alpha_{k}\right)\right\}=\left.\frac{\theta\left(q_{i-}\right)}{\left(2 q_{i-}\right)^{2}} \frac{\partial}{\partial q_{+}}\left(\prod_{j \neq i} G_{F}\left(q_{j}\right)\right) N\left(\alpha_{k}\right)\right|_{q_{i+}=q_{i+}^{(+)}} \\
=\int d q_{+} \delta_{+}\left(q_{i}^{2}-m_{i}^{2}\right)\left(\prod_{j \neq i} G_{D}\left(q_{i} ; q_{j}\right)\right)\left[-\sum_{j \neq i} \frac{\eta q_{j}}{\eta q_{i}} G_{D}\left(q_{i} ; q_{j}\right)+\frac{1}{2 \eta q_{i}} \frac{\partial}{\partial \eta q_{i}}\right] N\left(\alpha_{k}\right),
\end{gathered}
$$


where now $\eta^{\mu}=\left(\eta_{+}, \eta_{-}=0, \mathbf{0}_{\perp}\right)$. Eq. (3.10) has the same functional form as in eq. (3.6), although with a different dual vector $\eta$. Thus we can generalize eq. (3.6) and eq. (3.10) to an arbitrary coordinate system and combining simple and double poles in a single formula we get in cartesian coordinates:

$$
\begin{aligned}
\int_{q} G_{F}^{2}\left(q_{i}\right) & \left(\prod_{j \neq i} G_{F}\left(q_{j}\right)\right) N\left(\alpha_{k}\right)= \\
& -\int_{q}\left\{\tilde{\delta}\left(q_{i}\right)\left(\prod_{j \neq i} G_{D}\left(q_{i} ; q_{j}\right)\right)\left[-\frac{\eta^{2}}{2\left(\eta q_{i}\right)^{2}}-\sum_{n \neq i} \frac{\eta q_{j}}{\eta q_{i}} G_{D}\left(q_{i} ; q_{j}\right)+\frac{1}{2 \eta q_{i}} \frac{\partial}{\partial \eta q_{i}}\right]\right. \\
& \left.+\sum_{j \neq i} \tilde{\delta}\left(q_{j}\right) G_{D}^{2}\left(q_{j} ; q_{i}\right)\left(\prod_{k \neq i, j} G_{D}\left(q_{j} ; q_{k}\right)\right)\right\} N\left(\alpha_{k}\right) .
\end{aligned}
$$

Equation (3.11), is the main result of this section. It extends the Duality Theorem to integrals with identical propagators or, to put it differently, with double poles in the complex plane. For the case of the generic two-loop graph in figure 3, this result can be seen as an extension of eq. (2.15). If we have two groups of momenta, $\alpha_{k}, \alpha_{2}$, one of which contains the double propagator, i.e. $\alpha_{k}=\left\{q_{n}=\ell_{1}+\ell_{2}\right\}$ and $\alpha_{2}=$ $\left\{q_{2}=\ell_{2}, q_{3}=\ell_{2}+p_{1}, \ldots, q_{n-1}=\ell_{2}+p_{1, N-1}, q_{2}=\ell_{2}\right\}$, and we denote by $\alpha_{2}^{\prime}$ a group that contains all the momenta of $\alpha_{2}$ leading to single poles, namely

$\alpha_{2}^{\prime}=\left\{q_{2}=\ell_{2}, q_{3}=\ell_{2}+p_{1}, \ldots, q_{n-1}=\ell_{2}+p_{1, N-1}\right\}$, then we can write:

$$
\begin{aligned}
G_{D}\left(\alpha_{k} \cup \alpha_{2}\right)= & \tilde{\delta}\left(q_{2}\right)\left(\prod_{j \in \alpha_{2}^{\prime}, \alpha_{k}}^{n} G_{D}\left(q_{2} ; q_{j}\right)\right)\left[-\frac{\eta^{2}}{2\left(\eta q_{2}\right)^{2}}-\sum_{j \in \alpha_{2}^{\prime}, \alpha_{k}}^{n} \frac{\eta q_{j}}{\eta q_{2}} G_{D}\left(q_{2} ; q_{j}\right)\right] \\
& +\sum_{i \in \alpha_{2}^{\prime}, \alpha_{k}}^{n} \tilde{\delta}\left(q_{i}\right) G_{D}^{2}\left(q_{i} ; q_{2}\right)\left(\prod_{j \neq i}^{n} G_{D}\left(q_{i} ; q_{j}\right)\right) .
\end{aligned}
$$

This result states that for the case of a double pole, one follows the usual procedure of cutting every propagator line once, including the double propagator, and transforming the rest of the propagators to dual propagators. A similar formula can be derived for the case of multiple (triple and higher) poles. The calculation of the residue of a multiple pole introduces, however, contributions with powers of dual propagators. In absence of a general transformation formula analogous to eq. (2.14), it is not possible to rewrite eq. (3.11) in terms of dual propagators whose dual $+i 0$ prescription depends on the external momenta only. For that reason, we will present in the next section a different strategy for dealing with higher order poles based on the reduction of the integral using Integration By Parts.

\section{Reducing to single poles with IBPs}

In this section, we discuss a different approach to the generalization of the Duality Theorem to higher order poles. We will use Integration By Parts (IBP) $[28,29]$ to reduce the integrals with multiple poles to ones with simple poles. We emphasize the fact the we do not need to 
reduce the integrals to a particular integral basis. We just need to reduce them "enough", so that the higher order poles disappear.

To give a short introduction to the method and establish our notation, let us consider a general $m$-loop scalar integral in $d$ dimensions, with $n$ denominators $D_{1}, \ldots, D_{n}$ raised to exponents $a_{1}, \ldots, a_{n}$ and external momenta $p_{1}, \ldots, p_{N}$ :

$$
\int_{\ell_{1}} \cdots \int_{\ell_{m}} \frac{1}{D_{1}^{a_{1}} \cdots D_{n}^{a_{n}}}
$$

If we notice that

$$
\int_{\ell_{1}} \cdots \int_{\ell_{m}} \frac{\partial}{\partial s^{\mu}} \frac{t^{\mu}}{D_{1}^{a_{1}} \cdots D_{n}^{a_{n}}}=0
$$

where $s^{\mu}=\ell_{1}^{\mu}, \ell_{2}^{\mu}, \ldots, \ell_{m}^{\mu}$, the integrand being a total derivative with respect to the loop momenta, we can find relations between scalar integrals with different exponents $a_{i}$. This will allow us to express integrals with exponents larger than one, in terms of simpler ones. In effect, we will be able to write integrals with multiple poles in terms of sums of integrals with simple poles. In the numerator of the integrand of eq. (4.2) we can use $t^{\mu}=\ell_{1}^{\mu}, \ldots, \ell_{m}^{\mu}, p_{1}^{\mu}, \ldots, p_{N}^{\mu}$, to obtain a system of equations that relate the various integrals. For simplicity, when refering to an IBP we will use the shorthand notation:

$$
\frac{\partial}{\partial s} \cdot t
$$

to denote eq. (4.2). The differentiation will raise or leave an exponent unchanged, while, contractions with the loop and external momenta in the numerator of the integrand, can be expressed in terms of the propagators to lower an exponent. Often times though, this is not possible, leaving scalar products of momenta in the numerator, which cannot be expressed in terms of denominators. These are called Irreducible Scalar Products (ISP). To simplify the notation, we will denote these ISPs appearing in the numerator of the integrand as additional factors, $D_{i j}=\ell_{i} \cdot p_{j}$, raised to a negative power $a_{k}$. We use the notation:

$$
F\left(a_{1} a_{2} \cdots a_{n}\right)=\int_{\ell_{1}} \int_{\ell_{2}} \frac{1}{D_{1}^{a_{1}} D_{2}^{a_{2}} \cdots D_{n}^{a_{n}}}
$$

to denote a generic two-loop integral with $n$ propagators raised to an arbitrary integer power, with $D_{i}=G_{F}^{-1}\left(q_{i}\right)=q_{i}^{2}-m_{i}^{2}+i 0$ and $q_{i}$ denotes any combination of external and loop momenta. In the following the prescription $+i 0$ for the propagators is understood. We will use the symbol $\mathbf{a}_{\mathbf{i}}^{+}$to denote the raising of the index $a_{i}$ by one i.e. $\mathbf{1}^{+} F\left(a_{1}, a_{2}, \cdots a_{n}\right)=$ $F\left(a_{1}+1, a_{2}, \ldots, a_{n}\right)$ and the symbol $\mathbf{a}_{\mathbf{i}}^{-}$to denote the lowering of the index $a_{i}$ by one i.e. $\mathbf{2}^{-} F\left(a_{1}, a_{2}, \cdots a_{n}\right)=F\left(a_{1}, a_{2}-1, \ldots, a_{n}\right)$. A combination of the two means that the operators apply at the same time i.e. $\mathbf{1}^{+} \mathbf{2}^{-} F\left(a_{1}, a_{2}, \cdots a_{n}\right)=F\left(a_{1}+1, a_{2}-1, \ldots, a_{n}\right)$. In the following we will use two automated codes, for the reduction, FIRE [27], a MATHEMATICA package for the reduction of integrals and REDUZE 2 [30], ${ }^{1}$ a package written in $\mathrm{C}++$, using GiNaC [31].

\footnotetext{
${ }^{1}$ Since the most obvious first approach seems to be to try to express the integrals with multiple poles in terms of the same integrals with only single poles, cf. eq. (4.6), we used, in addition to the "usual" version of REDUZE 2, in some cases a special patch for REDUZE 2 which provides a modification of its integral ordering
} 


\subsection{The case for two-loop diagrams}

The only generic two-loop scalar graph with $N$-legs and a double propagator is shown in figure 3. The simplest case is the two-point function with massless internal lines. The denominators are:

$$
D_{1}=\ell_{1}^{2}, D_{2}=\ell_{2}^{2}, D_{3}=\left(\ell_{2}+p\right)^{2}, D_{4}=\left(\ell_{1}+\ell_{2}\right)^{2}, \quad D_{5}=\ell_{1} \cdot p
$$

where $D_{5}$ is indeed an ISP. ${ }^{2}$ We stress the fact that the additional scalar product we use (in this case and the cases below) is not an additional linear propagator. We merely use it to reduce the original integrals with the algebraic packages FIRE and REDUZE 2. These linear factors do not appear as linear propagators in any of the cases considered, but, they might appear as numerators in the final reduced result, and thus do not affect applying the Duality theorem. In our notation, the integral we want to reduce is $F(12110)$ and to this end we use the six total derivatives

$$
\frac{\partial}{\partial \ell_{i}} \cdot \ell_{j}, \frac{\partial}{\partial \ell_{i}} \cdot p, i, j=1,2
$$

Applying these IBPs on $F\left(a_{1} a_{2} a_{3} a_{4} a_{5}\right)$ we get a system of recursive equations. Using specific values for the exponents $a_{i}$ we can solve this system and obtain $F(12110)$. For this particular case, we solve the system explicitely and the reader is referred to the appendix $\mathrm{A}$ for details. Finally we arrive at:

$$
F(12110)=\frac{-1+3 \epsilon}{(1+\epsilon) s} F(11110)
$$

where $s=p^{2}+i 0$, a result which contains only single poles and can be treated using the Duality Theorem [23]. For the rest of the cases below and in the three-loop case in the next section, we have used FIRE and REDUZE 2 to perform the reductions and check our results. For three external legs $p_{1}^{2}=p_{2}^{2}=0, p_{3}^{2}=\left(p_{1}+p_{2}\right)^{2}$ and massless internal lines, we have the denominators:

$$
\begin{aligned}
& D_{1}=\ell_{1}^{2}, \quad D_{2}=\ell_{2}^{2}, \quad D_{3}=\left(\ell_{2}+p_{1}\right)^{2}, \quad D_{4}=\left(\ell_{2}+p_{1}+p_{2}\right)^{2}, \quad D_{5}=\left(\ell_{1}+\ell_{2}\right)^{2}, \\
& D_{6}=\ell_{1} \cdot p_{1}, \quad D_{7}=\ell_{1} \cdot p_{2} \text {, }
\end{aligned}
$$

where, the last two are the ISPs that appear in this case. The integral we want to reduce is $F(1211100)$. We use eight IBPs:

$$
\frac{\partial}{\partial \ell_{i}} \cdot \ell_{j}, \frac{\partial}{\partial \ell_{i}} \cdot p_{j}, i, j=1,2
$$

in the final result. This modified version of REDUZE 2 delivered the results for the integrals in this desired form stated in the subsequent sections, while we used the normal version of the integral ordering for the remaining cases. Note that we also calculated explicitely the relations obtained from the modified version, in the easiest cases of the massless two- and three-loop integrals which can be built by insertion of the massless one-loop two-point function, and found agreement.

${ }^{2}$ For REDUZE 2 the corresponding propagator is added and used as input instead. 
A similar analysis to the one above, gives:

$$
F(1211100)=\frac{3 \epsilon}{(1+\epsilon) s} F(1111100)=-\frac{3(1-3 \epsilon)(2-3 \epsilon)}{\epsilon(1+\epsilon) s^{3}} F(1001100),
$$

where $s=\left(p_{1}+p_{2}\right)^{2}+i 0$, which, again contains only single poles and can be treated with the Duality Theorem.

The inclusion of masses does not affect the general picture of the reduction. It solely introduces numerators in some integrals after the reduction is done. But, as we have stressed already, the application of the Duality Theorem is not affected by numerators since it only operates on denominators [23]. As an illustrative example, let us consider the two-loop graph with two external legs and one massive loop (see figure 3 ). For the case of the left loop being massive (related to $\ell_{2}$ ), with mass $m$, the denominators involved are

$$
D_{1}=\ell_{1}^{2}, \quad D_{2}=\ell_{2}^{2}-m^{2}, \quad D_{3}=\left(\ell_{2}+p\right)^{2}-m^{2}, D_{4}=\left(\ell_{1}+\ell_{2}\right)^{2}-m^{2},
$$

with the addition of the irreducible scalar product

$$
D_{5}=\ell_{1} \cdot p
$$

needed to perform the reduction. Using the same IBPs of eq. (4.5), the result of the reduction, with FIRE is:

$$
\begin{aligned}
F(12110)= & \frac{(\epsilon-1)\left[-\epsilon s^{2}+2 m^{2}\left(9 \epsilon-2 \epsilon^{2}-3\right) s+4 m^{4}(-3+2 \epsilon)(-1+2 \epsilon)\right]}{2 \epsilon(2 \epsilon-1) m^{4} s\left(4 m^{2}-s\right)^{2}} F(00110) \\
& +\frac{A}{2 \epsilon(2 \epsilon-1) m^{4} s\left(4 m^{2}-s\right)^{2}} F(10110)-\frac{(\epsilon-1)}{2(2 \epsilon-1) m^{4} s} F(1-1110) \\
& -\frac{(\epsilon-1)^{2}\left(2 m^{2}-s\right)}{(2 \epsilon-1) m^{4} s\left(4 m^{2}-s\right)} F(01010)-\frac{(\epsilon-1)\left(4 \epsilon m^{2}+2 m^{2}-s\right)}{2(2 \epsilon-1) m^{4}\left(4 m^{2}-s\right)} F(01110) \\
& +\frac{2(\epsilon-1)\left(m^{2}-s\right)\left(10 \epsilon m^{2}-\epsilon s-3 m^{2}\right)}{\epsilon(2 \epsilon-1) m^{4} s\left(4 m^{2}-s\right)^{2}} F(1011-1),
\end{aligned}
$$

with $s=p^{2}+i 0$ and

$$
A=(1-\epsilon)\left[\epsilon s+2(3-8 \epsilon) m^{2}\right] s^{2}+2(1-2 \epsilon) m^{4}\left[2(3-4 \epsilon) m^{2}-(6-5 \epsilon) s\right] .
$$

The reduction generates two integrals with a numerator, namely

$$
\begin{aligned}
& F(1-1110)=\int_{\ell_{1}} \int_{\ell_{2}} \frac{\ell_{2}^{2}-m^{2}}{D_{1} D_{3} D_{4}}, \\
& F(1011-1)=\int_{\ell_{1}} \int_{\ell_{2}} \frac{\ell_{1} \cdot p}{D_{1} D_{3} D_{4}},
\end{aligned}
$$


but the double poles have now disappeared. The result with REDUZE 2 reads:

$$
\begin{aligned}
F(12110)= & -\frac{(\epsilon-1)\left(4 \epsilon m^{2}+2 m^{2}-s\right)}{2(2 \epsilon-1) m^{4}\left(4 m^{2}-s\right)} F(01110) \\
& +\frac{3(\epsilon-1)\left(8 \epsilon m^{4}-12 \epsilon m^{2} s+\epsilon s^{2}-4 m^{4}+4 m^{2} s\right)}{2 \epsilon(2 \epsilon-1) m^{4} s\left(4 m^{2}-s\right)^{2}} F(1-1110) \\
& +\frac{A}{2 \epsilon(2 \epsilon-1) m^{4} s\left(4 m^{2}-s\right)^{2}} F(10110) \\
& +\frac{(\epsilon-1)\left(8 \epsilon^{2} m^{2} s-2 \epsilon^{2} s^{2}-16 \epsilon m^{4}+6 \epsilon m^{2} s+\epsilon s^{2}+12 m^{4}-6 m^{2} s\right)}{2 \epsilon(2 \epsilon-1) m^{4} s\left(4 m^{2}-s\right)^{2}} F(01010),
\end{aligned}
$$

where $A$ is given by eq. (4.11). Despite the appearence of different integrals the two results are of course equivalent. This is because, the integrals $F(00110)$ and $F(01010)$, in the result obtained with FIRE, are identical (as can be seen by shifting the loop momenta), so the sum of their coefficients gives exactly the coefficient of the result obtained with REDUZE 2 . The same argument applies for the integrals $F(1011-1)$ and $F(1-1110)$. The appearence of the numerators does not affect the application of the Duality Theorem for integrals with single poles as was detailed in [23]. For the case of the right loop in figure 3 being massive (related to $\ell_{1}$ ), we have the denominators:

$$
D_{1}=\ell_{1}^{2}-m^{2}, D_{2}=\ell_{2}^{2}, D_{3}=\left(\ell_{2}+p\right)^{2}, \quad D_{4}=\left(\ell_{1}+\ell_{2}\right)^{2}-m^{2}, \quad D_{5}=\ell_{1} \cdot p .
$$

Using the IBPs from eq. (4.5), we get with FIRE:

$$
\begin{aligned}
F(12110)= & \frac{\left(32 \epsilon^{2} m^{4}+8 \epsilon^{2} m^{2} s+\epsilon^{2} s^{2}-32 \epsilon m^{4}-11 \epsilon m^{2} s-\epsilon s^{2}+6 m^{4}+3 m^{2} s\right)}{6 m^{4} s^{2}} F(10110) \\
& -\frac{(\epsilon-1)\left(16 \epsilon^{3} m^{2}+4 \epsilon^{3} s-20 \epsilon^{2} m^{2}-8 \epsilon m^{2}-7 \epsilon s+3 m^{2}+3 s\right)}{6(2 \epsilon-1)(2 \epsilon+1) m^{4} s^{2}} F(10010) \\
& -\frac{(\epsilon-1) \epsilon}{3 m^{4} s} F(1011-1)-\frac{(\epsilon-1)(2 \epsilon-1)}{2 m^{2} s} F(01110) \\
& -\frac{(\epsilon-1)\left(12 \epsilon m^{2}+\epsilon s-3 m^{2}\right)}{6 m^{4} s^{2}} F(1-1110) \\
& -\frac{(\epsilon-1)\left(6 \epsilon m^{2}+\epsilon s-3 m^{2}\right)}{6 m^{4} s} F(11100),
\end{aligned}
$$

and with REDUZE 2:

$$
\begin{aligned}
F(12110)= & -\frac{(\epsilon-1)\left(8 \epsilon m^{2}+\epsilon s-2 m^{2}\right)}{4 m^{4} s^{2}} F(1-1110) \\
& +\frac{64 \epsilon^{2} m^{4}+16 \epsilon^{2} m^{2} s+\epsilon^{2} s^{2}-64 \epsilon m^{4}-22 \epsilon m^{2} s-\epsilon s^{2}+12 m^{4}+6 m^{2} s}{12 m^{4} s^{2}} F(10110) \\
& -\frac{(\epsilon-1)\left(12 \epsilon m^{2}+\epsilon s-6 m^{2}\right)}{6 m^{4} s} F(11100) \\
& -\frac{(\epsilon-1)(2 \epsilon-3)\left(16 \epsilon^{2} m^{2}+2 \epsilon^{2} s+4 \epsilon m^{2}+3 \epsilon s-2 m^{2}-2 s\right)}{12(2 \epsilon-1)(2 \epsilon+1) m^{4} s^{2}} F(10010) .
\end{aligned}
$$


For the case of the double pole, two-loop graph, with three external legs and one massive loop, we have the denominators:

$$
\begin{array}{lll}
D_{1}=\ell_{1}^{2}, & D_{2}=\ell_{2}^{2}-m^{2}, & D_{3}=\left(\ell_{2}+p_{1}\right)^{2}-m^{2}, \\
D_{4}=\left(\ell_{2}+p_{1}+p_{2}\right)^{2}-m^{2}, & D_{5}=\left(\ell_{1}+\ell_{2}\right)^{2}-m^{2}, & \\
D_{6}=\ell_{1} \cdot p_{1}, & D_{7}=\ell_{1} \cdot p_{2} .
\end{array}
$$

Using the IBPs from eq. (4.7) we get with FIRE:

$$
\begin{aligned}
F(1211100)= & \frac{(\epsilon-1)\left\{(1+4 \epsilon) s^{2}-4 \epsilon m^{2} s(11-2 \epsilon)-8 m^{4}\left(4 \epsilon^{2}-8 \epsilon-1\right)\right\}}{8 \epsilon(2 \epsilon-1) m^{6} s\left(4 m^{2}-s\right)^{2}} F(0001100) \\
& -\frac{A_{1}}{2 \epsilon(2 \epsilon-1) m^{6} s\left(4 m^{2}-s\right)^{2}} F(1001100) \\
& +\frac{\left(8 \epsilon^{3}-12 \epsilon^{2}+4 \epsilon-1\right)(\epsilon-1)}{8 \epsilon(2 \epsilon-1)^{2} m^{6} s} F(0010100) \\
& -\frac{(\epsilon-1)}{2(2 \epsilon-1) m^{4}}[F(0111100)-F(1011100)]+\frac{2(\epsilon-1)}{m^{2} s\left(4 m^{2}-s\right)} F(0101100) \\
& +\frac{(\epsilon-1)\left(2 \epsilon m^{2}-\epsilon s-m^{2}\right)}{2 \epsilon(2 \epsilon-1) m^{6} s^{2}} F(1-101100) \\
& +\frac{(\epsilon-1)^{2}\left(8 \epsilon m^{2}-2 \epsilon s-6 m^{2}+s\right)}{2(2 \epsilon-1) m^{6} s\left(4 m^{2}-s\right)} F(0100100) \\
& -\frac{2(\epsilon-1)\left(m^{2}-s\right)\left\{-\epsilon s^{2}+m^{2} s(6 \epsilon-1)+8 m^{4}(2 \epsilon-1)\right\}}{\epsilon(2 \epsilon-1) m^{6} s^{2}\left(4 m^{2}-s\right)^{2}} F(10011-10),
\end{aligned}
$$

where $s=\left(p_{1}+p_{2}\right)^{2}+i 0$, and:

$$
A_{1}=\epsilon(1-\epsilon) s^{3}+m^{2}(-1+\epsilon)(-1+9 \epsilon) s^{2}+m^{4}(1-\epsilon)(5+6 \epsilon) s+2 m^{6}(1+2 \epsilon)(-3+4 \epsilon),
$$

while, with REDUZE 2, we get:

$$
\begin{aligned}
F(1211100)= & -\frac{\epsilon-1}{2(2 \epsilon-1) m^{4}} F(0111100) \\
& +\frac{\epsilon-1}{2(2 \epsilon-1) m^{4}} F(1011100) \\
& +\frac{2(\epsilon-1)}{m^{2} s\left(4 m^{2}-s\right)} F(0101100) \\
& -\frac{3(\epsilon-1)\left(4 \epsilon m^{4}-8 \epsilon m^{2} s+\epsilon s^{2}+2 m^{4}+m^{2} s\right)}{2 \epsilon(2 \epsilon-1) m^{6} s\left(4 m^{2}-s\right)^{2}} F(1-101100) \\
& -\frac{A_{2}}{2 \epsilon(2 \epsilon-1) m^{6} s\left(4 m^{2}-s\right)^{2}} F(1001100) \\
& +\frac{A_{3}}{4 \epsilon(2 \epsilon-1)^{2} m^{6} s\left(4 m^{2}-s\right)^{2}} F(0100100)
\end{aligned}
$$


where

$$
\begin{aligned}
A_{2}= & 16 \epsilon^{2} m^{6}-6 \epsilon^{2} m^{4} s+9 \epsilon^{2} m^{2} s^{2}-\epsilon^{2} s^{3} \\
& -4 \epsilon m^{6}+\epsilon m^{4} s-10 \epsilon m^{2} s^{2}+\epsilon s^{3}-6 m^{6}+5 m^{4} s+m^{2} s^{2},
\end{aligned}
$$

and

$$
\begin{aligned}
A_{3}= & (\epsilon-1)\left(128 \epsilon^{4} m^{4}-64 \epsilon^{4} m^{2} s+8 \epsilon^{4} s^{2}-256 \epsilon^{3} m^{4}+112 \epsilon^{3} m^{2} s-12 \epsilon^{3} s^{2}+192 \epsilon^{2} m^{4}\right. \\
& \left.-92 \epsilon^{2} m^{2} s+8 \epsilon^{2} s^{2}-40 \epsilon m^{4}+26 \epsilon m^{2} s-\epsilon s^{2}-12 m^{4}+4 m^{2} s-s^{2}\right)
\end{aligned}
$$

The cases with additional external legs can be treated in a similar manner. It can always be reduced to sums of integrals with single propagators at the expense of introducing numerators. In fact in all the cases we studied so far (also for the three-loop cases below) it has been possible to reduce integrals with multiple propagators to integrals where only single poles appear (see also ref. [22] where a similar strategy is used to to cancel double propagators).

Our strategy is now clear. For a two-loop calculation, first we reduce all double pole graphs using IBPs or any other method. The remaining integrals all contain single poles and can be treated using the Duality Theorem at two-loops. The appearence of vector or tensor integrals does not spoil this strategy since the Duality Theorem for single poles, affects only the denominators of the integrands.

\subsection{The case for three-loop diagrams}

For three-loop graphs there exists one topology with a triple propagator and a number of topologies with a double propagator. All topologies are shown in figure 4. The arguments for the two-loop case are valid here as well. We first reduce the multiple pole integrands by using IBPs until we have integrals with only single poles (possibly with numerators) and then we can then apply single-pole Duality Theorem as it was described for the three-loop case in ref. [23]. In the following, we show explicitly the reduction of the two-point function for the different topologies and for massless internal lines. We use the notation:

$$
F\left(a_{1} a_{2} \cdots a_{n}\right)=\int_{\ell_{1}} \int_{\ell_{2}} \int_{\ell_{3}} \frac{1}{D_{1}^{a_{1}} D_{2}^{a_{2}} \cdots D_{n}^{a_{n}}}
$$

to denote a generic three-loop integral with $n$ propagators raised to an arbitrary integer power, with $D_{i}=G_{F}^{-1}\left(q_{i}\right)=q_{i}^{2}-m_{i}^{2}+i 0$ or $D_{i}$ equal to any ISP and $q_{i}$ any combination of external and loop momenta. For the rest of this section the prescription $+i 0$ for the propagators is understood. We also have $s=p^{2}+i 0$. The IBPs to be used for the reduction are:

$$
\frac{\partial}{\partial \ell_{i}} \cdot \ell_{j}, \frac{\partial}{\partial \ell_{i}} \cdot p, \quad i, j=1,2,3
$$

In the following, we present first the result obtained with REDUZE 2 and then with FIRE. For the single triple pole graph (a) in figure (4), we have the following expressions: 


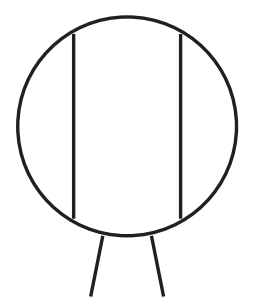

(a)

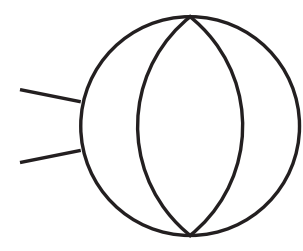

(d)

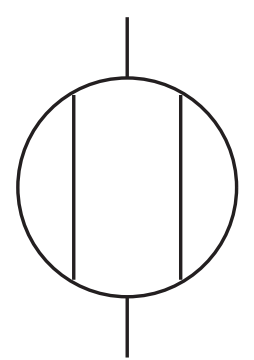

(b)

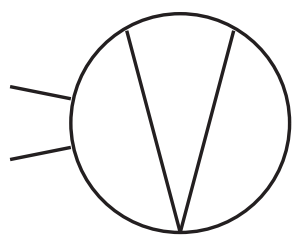

(e)

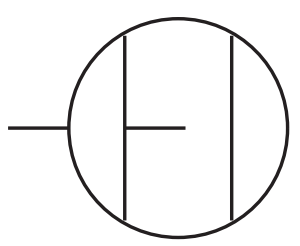

(g)

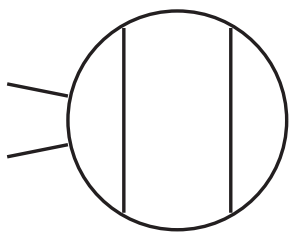

(c)

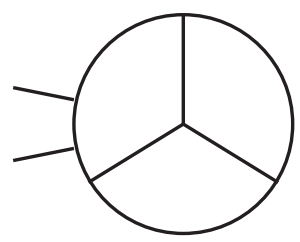

(f) agators. Each internal line already dressed with some external leg can be dressed with additional external lines.

(a) The denominators used are:

$$
\begin{aligned}
& D_{1}=\ell_{1}^{2}, \quad D_{2}=\ell_{2}^{2}, \quad D_{3}=\ell_{3}^{2}, \quad D_{4}=\left(\ell_{2}-p\right)^{2}, \quad D_{5}=\left(\ell_{1}-\ell_{2}\right)^{2}, \quad D_{6}=\left(\ell_{3}-\ell_{2}\right)^{2}, \\
& D_{7}=\ell_{1} \cdot p, \quad D_{8}=\ell_{3} \cdot p, \quad D_{9}=\ell_{1} \cdot \ell_{3} \text {, }
\end{aligned}
$$

with the result:

$$
\begin{aligned}
F(131111000) & =\frac{2 \epsilon(-1+4 \epsilon)}{(1+\epsilon)(1+2 \epsilon) s^{2}} F(111111000) \\
& =\frac{2(-1+2 \epsilon)(-1+4 \epsilon)}{(1+\epsilon)(1+2 \epsilon) s^{3}} F(101111000) .
\end{aligned}
$$

For the graphs with doubles poles, (b)-(g), figure (4), we find:

(b) The denominators are:

$$
\begin{aligned}
& D_{1}=\ell_{1}^{2}, \quad D_{2}=\ell_{2}^{2}, \quad D_{3}=\ell_{3}^{2}, \quad D_{4}=\left(\ell_{2}-p\right)^{2}, \quad D_{5}=\left(\ell_{1}-\ell_{2}\right)^{2}, \quad D_{6}=\left(\ell_{3}-\ell_{2}+p\right)^{2}, \\
& D_{7}=\ell_{1} \cdot p, \quad D_{8}=\ell_{1} \cdot \ell_{3}, \quad D_{9}=\ell_{3} \cdot p \text {, }
\end{aligned}
$$


with the result:

$$
\begin{aligned}
F(121211000) & =\frac{3(-1+4 \epsilon)(1+3 \epsilon)}{(1+\epsilon)^{2} s^{2}} F(111111000) \\
& =\frac{6(-2+3 \epsilon)(-1+3 \epsilon)(1+3 \epsilon)(-3+4 \epsilon)(-1+4 \epsilon)}{\epsilon^{2}(1+\epsilon)^{2}(-1+2 \epsilon) s^{4}} F(101011000) .
\end{aligned}
$$

(c) The denominators are:

$$
\begin{aligned}
& D_{1}=\ell_{1}^{2}, \quad D_{2}=\ell_{2}^{2}, \quad D_{3}=\ell_{3}^{2}, \quad D_{4}=\left(\ell_{3}+p\right)^{2}, \quad D_{5}=\left(\ell_{3}-\ell_{2}\right)^{2}, \quad D_{6}=\left(\ell_{1}-\ell_{2}\right)^{2}, \\
& D_{7}=\ell_{1} \cdot p, \quad D_{8}=\ell_{2} \cdot p, \quad D_{9}=\ell_{1} \cdot \ell_{3},
\end{aligned}
$$

with the result:

$$
\begin{aligned}
F(122111000) & =\frac{2 \epsilon(-1+4 \epsilon)(-1+3 \epsilon)}{(1+2 \epsilon)(1+\epsilon)^{2} s^{2}} F(111111000) \\
& =\frac{2(-2+3 \epsilon)(-1+3 \epsilon)(-3+4 \epsilon)(-1+4 \epsilon)}{\epsilon(1+\epsilon)^{2}(1+2 \epsilon) s^{4}} F(100111000)
\end{aligned}
$$

(d) The denominators are:

$$
\begin{array}{lllll}
D_{1}=\ell_{1}^{2}, & D_{2}=\ell_{2}^{2}, & D_{3}=\ell_{3}^{2}, & D_{4}=\left(\ell_{3}-p\right)^{2}, & D_{5}=\left(\ell_{2}+\ell_{3}-\ell_{1}\right)^{2}, \\
D_{6}=\ell_{1} \cdot p, & D_{7}=\ell_{2} \cdot p, & D_{8}=\ell_{1} \cdot \ell_{2}, & D_{9}=\ell_{1} \cdot \ell_{3},
\end{array}
$$

with the result:

$$
F(112110000)=\frac{(-1+2 \epsilon)}{\epsilon s} F(111110000)=\frac{(-3+4 \epsilon)}{\epsilon s^{2}} F(110110000) .
$$

(e) The denominators are:

$$
\begin{array}{cllll}
D_{1}=\ell_{1}^{2}, & D_{2}=\ell_{2}^{2}, & D_{3}=\ell_{3}^{2}, & D_{4}=\left(\ell_{3}-p\right)^{2}, & D_{5}=\left(\ell_{1}+\ell_{2}\right)^{2}, \quad D_{6}=\left(\ell_{2}+\ell_{3}\right)^{2}, \\
& D_{7}=\ell_{1} \cdot p, & D_{8}=\ell_{1} \cdot \ell_{3}, & D_{9}=\ell_{2} \cdot p,
\end{array}
$$

with the result:

$$
F(112111000)=\frac{(-1+4 \epsilon)}{(1+2 \epsilon) s} F(111111000)=\frac{(-2+3 \epsilon)(-3+4 \epsilon)(-1+4 \epsilon)}{\epsilon^{2}(1+2 \epsilon) s^{3}} F(100111000) .
$$

(f) The denominators are:

$$
\begin{aligned}
& D_{1}=\ell_{1}^{2}, \quad D_{2}=\ell_{2}^{2}, \quad D_{3}=\ell_{3}^{2}, \quad D_{4}=\left(\ell_{2}+p\right)^{2}, \quad D_{5}=\left(\ell_{1}+\ell_{2}\right)^{2}, \quad D_{6}=\left(\ell_{1}+\ell_{3}\right)^{2}, \\
& D_{7}=\left(\ell_{3}-\ell_{2}\right)^{2}, \quad D_{8}=\ell_{1} \cdot p, \quad D_{9}=\ell_{3} \cdot p,
\end{aligned}
$$

with the result:

$$
\begin{aligned}
F(121111100) & =\frac{2 \epsilon}{(1+\epsilon) s} F(111111100) \\
= & \frac{2(-2+3 \epsilon)(-1+3 \epsilon)(-3+4 \epsilon)(-1+4 \epsilon)}{\epsilon^{2}(1+\epsilon)(1+2 \epsilon) s^{4}}[F(001111000)+F(100101100)] \\
& +\frac{2(-1+2 \epsilon)^{2}(-1+4 \epsilon)}{\epsilon(1+\epsilon)(1+2 \epsilon) s^{3}} F(101110100) .
\end{aligned}
$$


(g) The denominators are:

$$
\begin{array}{llll}
D_{1}=\ell_{1}^{2}, & D_{2}=\ell_{2}^{2}, & D_{3}=\ell_{3}^{2}, & D_{4}=\left(\ell_{3}-p\right)^{2}, \\
D_{5}=\left(\ell_{1}-\ell_{2}\right)^{2}, & D_{6}=\left(\ell_{3}-\ell_{2}\right)^{2}, & D_{7}=\left(\ell_{3}-\ell_{2}-p\right)^{2} & \\
D_{8}=\left(\ell_{1}-\ell_{3}\right)^{2}, & D_{9}=\left(\ell_{1}-p\right)^{2} & &
\end{array}
$$

with the result:

$$
\begin{aligned}
F(121111100)= & \frac{(-1+3 \epsilon)^{2}(1+5 \epsilon)}{(1+\epsilon)(1+2 \epsilon)^{2} s^{2}} F(1111111-10) \\
& +\frac{\epsilon\left(9 \epsilon^{2}-11 \epsilon-4\right)}{(1+\epsilon)(1+2 \epsilon)^{2} s^{2}} F(111111100) .
\end{aligned}
$$

The difference between the two results is due to the fact that the second is expressed in terms of basis integrals while the first is expressed in terms of integrals with single poles of the same type as the multiple pole integral (in effect the first result can be further reduced to the second). Since we do not seek a particular basis for our reduction, as was stressed earlier, both results are equally useful as far as application of the Duality Theorem is concerned.

\section{Conclusions}

We have extended the Duality Theorem to two- and three-loop integrals with multiple poles. A Lorentz invariant expression for the residues of double poles has been derived, which can be extended straightforwardly to triple and, in general, multiple poles. In the absence of a systematic procedure to express the dual $+i 0$ prescription in terms of external momenta exclusively, as in the case of single poles, we have explored an alternative approach. We use IBP identities to reduce the integrals with identical propagators to ones with only single poles. Therefore, the essential features of the loop-tree duality now remain intact. We reiterate that our goal is not to reduce everything to some set of master integrals. Rather, we reduce the integrals until there are no multiple poles left. Then, we can use the Duality Theorem in its original form for single pole propagators, to rewrite them as integrals of a tree-level object over a modified phase-space. The appearence of additional tensor integrals, due to the reduction, does not affect our procedure, since applying the Duality Theorem in its single-pole version, only cuts propagators, leaving the numerators of the integrals unaffected.

\section{Acknowledgments}

This work has been supported in part by the Spanish Government and ERDF funds from the EU Commission [grants FPA2007-60323, FPA2011-23778, CSD2007-00042 (Consolider Project CPAN)] and by GV (PROMETEO/2008/069), by the German Federal Ministry for Education and Research BMBF through Grant No. 05 HT6GUA by the Helmholtz Association HGF through Grant No. Ha 101 and by the German Research Foundation 
DFG through the Collaborative Research Center No 676 Particles, Strings and the Early Universe-The Structure of Matter and Space-Time. This work was also supported by the Research Executive Agency (REA) of the European Union under the Grant Agreement number PITN-GA-2010-264564 (LHCPhenoNet). We would like to thank Stefano Catani for many elucidating discussions. I.B. would like to thank Andreas von Manteuffel for providing us with the patch for REDUZE 2 that modified its integral ordering for our purposes.

\section{A Proof of the reduction of eq. (4.6)}

Here we solve the system of equations, explicitely, to arrive at eq. (4.6). We note that we are not aiming for a full reduction to a set of master integrals but rather to reduce the multiple poles to single poles. Therefore, any integral which has single propagators is to be considered known.

Using the IBPs, eq. (4.5), on the generic integral $F\left(a_{1} a_{2} a_{3} a_{4} a_{5}\right)$, we get the system of equations:

$$
\begin{aligned}
& d-2 a_{1}-a_{4}-a_{5}-a_{4} \mathbf{4}^{+} \mathbf{1}^{-}+a_{4} \mathbf{4}^{+} \mathbf{2}^{-}=0, \\
& a_{1}-a_{4}+\frac{1}{2} s a_{5} \mathbf{5}^{+}+a_{4} \mathbf{4}^{+}\left(\mathbf{1}^{-}-\mathbf{2}^{-}\right)+a_{1} \mathbf{1}^{+}\left(\mathbf{2}^{-}-\mathbf{4}^{-}\right)+\frac{1}{2} a_{5} \mathbf{5}^{+}\left(\mathbf{2}^{-}-\mathbf{3}^{-}\right)=0, \\
& a_{4} \mathbf{4}^{+}\left(s+\mathbf{2}^{-}-\mathbf{3}^{-}-2 \mathbf{5}^{-}\right)-s a_{5} \mathbf{5}^{+}-2 a_{1} \mathbf{1}^{+} \mathbf{5}^{-}=0, \\
& a_{2}-a_{4}+a_{2} \mathbf{2}^{+}\left(\mathbf{1}^{-}-4^{-}\right)+a_{3} 3^{+}\left(\mathbf{1}^{-}+\mathbf{2}^{-}-4^{-}-2 \mathbf{5}^{-}\right)+a_{4} \mathbf{4}^{+}\left(\mathbf{2}^{-}-\mathbf{1}^{-}\right)=0, \\
& d-2 a_{2}-a_{3}-a_{4}+a_{3} \mathbf{3}^{+}\left(s-\mathbf{2}^{-}\right)+a_{4} \mathbf{4}^{+}\left(\mathbf{1}^{-}-\mathbf{2}^{-}\right)=0, \\
& a_{2}-a_{3}+s\left(a_{2} \mathbf{2}^{+}-a_{3} \mathbf{3}^{+}+a_{4} \mathbf{4}^{+}\right)+a_{4} \mathbf{4}^{+}\left(\mathbf{2}^{-}-\mathbf{3}^{-}-2 \mathbf{5}^{-}\right)+a_{3} \mathbf{3}^{+} \mathbf{2}^{-}-a_{2} \mathbf{2}^{+} \mathbf{3}^{-}=0,
\end{aligned}
$$

where $s=p^{2}+i 0$. The appearence of the operator $5^{-}$signals that we have the ISP $\ell_{1} \cdot p$ in the numerator of an integral. As long as these integrals possess single propagators, we will not reduce them further but consider them known. We also note that a lot of the integrals that appear after setting particular values to the parameters $a_{i}$ in this system, are zero in dimensional regularization (in the massless case). Let us start by setting $a_{2}=2, a_{1}=a_{3}=$ $a_{4}=1, a_{5}=-1$ in (A.2). We get:

$$
F(2111-1)-F(1112-1)-\frac{1}{2} s F(12110)-\frac{1}{2} F(11110)=0 .
$$

Taking the sum of (A.5) and (A.6) and setting $a_{1}=1, a_{2}=a_{3}=a_{4}=1, a_{5}=0$ we get:

$$
(d-4) F(11110)+s F(12110)+s F(11120)-2 F(1112-1)=0 .
$$

Taking the difference between (A.5) and (A.6) and setting $a_{1}=2, a_{2}=a_{3}=a_{4}=1, a_{5}=0$ we get:

$$
-2 F(2111-1)-s F(12110)+s F(11210)-F(10210)=0 .
$$


Finally, setting $a_{1}=2, a_{2}=a_{3}=a_{4}=1, a_{5}=-1$ in (A.1), we get:

$$
(d-4) F(2111-1)-F(1112-1)+F(2012-1)=0 .
$$

The integrals $F(10120)$ and $F(11210)$ and $F(2012-1)$, in this system of equations, can be computed simply by taking further, appropriate combinations of eqs. (A.1)-(A.6). Setting $a_{1}=a_{2}=a_{3}=a_{4}=1, a_{5}=0$ in (A.1) we get $F(10120)=(3-d) F(11110)$. It also holds that $F(10120)=F(10210)$, as can be seen by making the shifts in the loop momenta $\ell_{2} \rightarrow-\ell_{1}-\ell_{2}-p$. From eq. (A.5), by setting $a_{1}=a_{2}=a_{3}=a_{4}=1, a_{5}=0$ and using the value of $F(10120)$, we get: $s F(11210)=(10-3 d) F(11110)$. Finally, adding eqs. (A.1) and (A.2) and setting $a_{1}=a_{2}=1, a_{3}=2, a_{4}=1, a_{5}=-1$ we get: $F(2012-1)=$ $(d-3)(d-4) F(11110)$. The rest of the system of equations (A.7)-(A.10) can now be solved sequencially, arriving at:

$$
F(12110)=\frac{(3 d-10)}{(d-6) s} F(11110),
$$

a result which, after putting $d=4-2 \epsilon$, agrees with eq. (4.6).

\section{References}

[1] C. Berger, Z. Bern, L.J. Dixon, F. Febres Cordero, D. Forde, et al., Precise predictions for $W+3$ jet production at hadron colliders, Phys. Rev. Lett. 102 (2009) 222001 [arXiv:0902.2760] [INSPIRE].

[2] K. Melnikov and G. Zanderighi, W+3 jet production at the LHC as a signal or background, Phys. Rev. D 81 (2010) 074025 [arXiv:0910.3671] [INSPIRE].

[3] G. Bevilacqua, M. Czakon, C. Papadopoulos, R. Pittau and M. Worek, Assault on the NLO wishlist: $p p \rightarrow t \bar{t} b \bar{b}, J H E P 09$ (2009) 109 [arXiv:0907.4723] [INSPIRE].

[4] A. Bredenstein, A. Denner, S. Dittmaier and S. Pozzorini, NLO QCD corrections to top anti-top bottom anti-bottom production at the LHC: 2. Full hadronic results, JHEP 03 (2010) 021 [arXiv: 1001.4006] [INSPIRE].

[5] P. Bolzoni, F. Maltoni, S.-O. Moch and M. Zaro, Higgs production via vector-boson fusion at NNLO in QCD, Phys. Rev. Lett. 105 (2010) 011801 [arXiv:1003.4451] [INSPIRE].

[6] S. Catani, G. Ferrera and M. Grazzini, $W$ boson production at hadron colliders: the lepton charge asymmetry in NNLO QCD, JHEP 05 (2010) 006 [arXiv: 1002.3115] [INSPIRE].

[7] S. Catani, L. Cieri, G. Ferrera, D. de Florian and M. Grazzini, Vector boson production at hadron colliders: a fully exclusive QCD calculation at NNLO,

Phys. Rev. Lett. 103 (2009) 082001 [arXiv:0903.2120] [INSPIRE].

[8] C. Anastasiou, G. Dissertori and F. Stockli, NNLO QCD predictions for the $H \rightarrow W W \rightarrow l \nu l \nu$ signal at the LHC, JHEP 09 (2007) 018 [arXiv:0707.2373] [INSPIRE].

[9] C. Anastasiou, K. Melnikov and F. Petriello, Fully differential Higgs boson production and the di-photon signal through next-to-next-to-leading order, Nucl. Phys. B 724 (2005) 197 [hep-ph/0501130] [INSPIRE]. 
[10] K. Melnikov and F. Petriello, Electroweak gauge boson production at hadron colliders through $o\left(\alpha_{s}^{2}\right)$, Phys. Rev. D 74 (2006) 114017 [hep-ph/0609070] [INSPIRE].

[11] S. Catani and M. Grazzini, An NNLO subtraction formalism in hadron collisions and its application to Higgs boson production at the LHC, Phys. Rev. Lett. 98 (2007) 222002 [hep-ph/0703012] [INSPIRE].

[12] S. Catani, L. Cieri, D. de Florian, G. Ferrera and M. Grazzini, Diphoton production at hadron colliders: a fully-differential QCD calculation at NNLO,

Phys. Rev. Lett. 108 (2012) 072001 [arXiv:1110.2375] [INSPIRE].

[13] A. Gehrmann-De Ridder, T. Gehrmann, E. Glover and G. Heinrich, Infrared structure of $e^{+} e^{-} \rightarrow 3$ jets at NNLO, JHEP 11 (2007) 058 [arXiv:0710.0346] [INSPIRE].

[14] A. Gehrmann-De Ridder, T. Gehrmann, E. Glover and G. Heinrich, NNLO corrections to event shapes in $e^{+} e^{-}$annihilation, JHEP 12 (2007) 094 [arXiv:0711.4711] [INSPIRE].

[15] S. Weinzierl, NNLO corrections to 3-jet observables in electron-positron annihilation, Phys. Rev. Lett. 101 (2008) 162001 [arXiv:0807.3241] [INSPIRE].

[16] P. Baernreuther, M. Czakon and A. Mitov, Percent level precision physics at the Tevatron: first genuine NNLO QCD corrections to $q \bar{q} \rightarrow t \bar{t}+x$, Phys. Rev. Lett. 109 (2012) 132001 [arXiv: 1204.5201] [INSPIRE].

[17] SM and NLO Multileg Working Group collaboration, J. Andersen et al., The SM and NLO multileg working group: summary report, arXiv:1003.1241 [INSPIRE].

[18] A. Denner, S. Dittmaier, T. Kasprzik and A. Muck, Electroweak corrections to $W+$ jet hadroproduction including leptonic W-boson decays, JHEP 08 (2009) 075 [arXiv:0906.1656] [INSPIRE].

[19] J.H. Kuhn, A. Kulesza, S. Pozzorini and M. Schulze, Electroweak corrections to hadronic production of $W$ bosons at large transverse momenta, Nucl. Phys. B 797 (2008) 27 [arXiv:0708.0476] [INSPIRE].

[20] S. Catani, T. Gleisberg, F. Krauss, G. Rodrigo and J.-C. Winter, From loops to trees by-passing Feynman's theorem, JHEP 09 (2008) 065 [arXiv:0804.3170] [INSPIRE].

[21] S. Caron-Huot, Loops and trees, JHEP 05 (2011) 080 [arXiv: 1007.3224] [INSPIRE].

[22] J. Gluza, K. Kajda and D.A. Kosower, Towards a basis for planar two-loop integrals, Phys. Rev. D 83 (2011) 045012 [arXiv: 1009.0472] [INSPIRE].

[23] I. Bierenbaum, S. Catani, P. Draggiotis and G. Rodrigo, A tree-loop duality relation at two loops and beyond, JHEP 10 (2010) 073 [arXiv:1007.0194] [INSPIRE].

[24] I. Bierenbaum, Towards a loop-tree duality at two loops and beyond, Nucl. Phys. Proc. Suppl. 205-206 (2010) 164 [arXiv:1007.0213] [InSPIRE].

[25] R. Feynman, Quantum theory of gravitation, Acta Phys. Polon. 24 (1963) 697 [InSPIRE].

[26] R.P. Feynman, Closed loop and tree diagrams, in Magic without magic, J.R. Klauder ed., Freeman, San Francisco U.S.A. (1972) 355 [in Selected papers of Richard Feynman, L.M. Brown ed., World Scientific, Singapore (2000) 867].

[27] A. Smirnov, Algorithm FIRE - Feynman integral reduction, JHEP 10 (2008) 107 [arXiv:0807.3243] [INSPIRE]. 
[28] K. Chetyrkin and F. Tkachov, Integration by parts: the algorithm to calculate $\beta$-functions in 4 loops, Nucl. Phys. B 192 (1981) 159 [InSPIRE].

[29] V.A. Smirnov, Feynman integral calculus, Springer-Verlag, Berlin Germany (2006).

[30] A. von Manteuffel and C. Studerus, Reduze 2 - Distributed Feynman integral reduction, arXiv: 1201.4330 [INSPIRE].

[31] C. Bauer, A. Frink, R. Kreckel Introduction to the GiNaC framework for symbolic computation within the C++ programming language, J. Symb. Comp. 33 (2002) 1 [http://www.ginac.de] [cs/0004015]. 